Pathologe 2012 · 33:496-507

DOI 10.1007/s00292-012-1649-x

Online publiziert: 16. September 2012

c) Springer-Verlag Berlin Heidelberg 2012

Schwerpunktherausgeber

F. Fend, Tübingen

Die histologische Knochenmarkuntersuchung ist ein essenzieller Bestandteil der Diagnostik hämatologischer und auch nichthämatologischer Krankheiten [12]. Diese besteht einerseits aus der Beurteilung der knöchernen Bestandteile und andererseits des Knochenmarks. Das normale Knochenmark des Erwachsenen setzt sich aus Fettgewebe und blutbildenden Zellen zusammen, die beim Erwachsenen im Axialskelett sowie in den proximalen Abschnitten der langen Röhrenknochen aufzufinden sind [12]. Bevorzugt wird, wegen der relativ komplikationslosen Entnahmemöglichkeit, die Knochenmarkuntersuchung vom hinteren Beckenkamm [1]. Für die histologische Untersuchung sind das Alter des Patienten, der klinische Zusammenhang (Fragestellung, Vorerkrankung, Blutbild) sowie die (Medikamenten-) Anamnese die wichtigsten Informationen, ohne die eine histologische Knochenmarkdiagnostik nur unvollständig möglich ist.

\section{Das normale Knochenmark}

\section{Knöcherne Bestandteile}

Diese bestehen aus Kompakta und Spongiosa (Knochenbälkchen) mit den entsprechenden Knochenzellen, Osteoblasten, -klasten und -zyten. Neben Knochenumbau wird das Volumen des spongiösen Knochens und der Kompakta beurteilt, wobei dies ohne morphometrische

A. Tzankov' ${ }^{1}$ S. Dirnhofer ${ }^{1}$. C. Beham-Schmid ${ }^{1}$

${ }^{1}$ Institut für Pathologie, Universitätsspital Basel, Schweiz

${ }^{2}$ Institut für Pathologie, Medizinische Universität Graz, Österreich

\title{
Normales Knochenmark und häufige reaktive Veränderungen
}

Hilfsmittel, subjektiv, semiquantitativ erfolgt. Im kindlichen Knochenmark zeigt sich lebhafter Knochenumbau, während im Erwachsenenknochen so gut wie kein Umbau vorhanden ist [39].

\section{Markzellularität}

Unter Markzellularität versteht man die relative Menge von Hämatopoiese und Fettgewebe, wobei die Normwerte mit individuellen Schwankungen altersabhängig sind $[12,34]$. Am Schnittpräparat gibt man die Zellularität als Flächenprozentwert an, d. h. wie viel Prozent von hämatopoietischen Zellen eingenommen werden. Diese Prozentwerte können auf das gesamte Biopsat einschließlich des Knochengewebes bezogen werden, oder, ähnlich der Aspiratuntersuchung, auf den Markrauminhalt ohne Spongiosa [1].

Beim Neugeborenen ist das Knochenmark zellreich mit einer zu vernachlässigenden Anzahl von Fettzellen. Die Zellularität vermindert sich mit zuneh- mendem Lebensalter gleichmäßig, wobei ab dem 70. Lebensjahr ein beschleunigter Abfall der Zellularität zu beobachten ist. Durchschnittlich findet man bei 20-Jährigen $70-80 \%$, bei 50 -Jährigen etwa $50-60 \%$ und bei Menschen im Alter von mehr als 70 Jahren $20-30 \%$ zelluläres Mark (• Abb. 1; $[15,34])$.

Der häufigste diagnostische Irrtum bei der Beurteilung der Zellularität ist ein räumlicher Zellularitätsunterschied in Form des sog. subkortikalen Fettgürtels (- Abb. 2a). Vermeiden kann man diesen Fehler, wenn man die knöchernen Strukturen im Biopsat beachtet (z. B. tangentiale Entnahme mit reichlich miterfasster Kortikalis) und ausreichend Markräume zur Beurteilung vorliegen hat. $\mathrm{Zu}$ mindest drei intakte intertrabekuläre Markraumareale sollten zur Beurteilung vorliegen [40].
Abb. 1 Altersabhängige Zellularität im Knochenmark

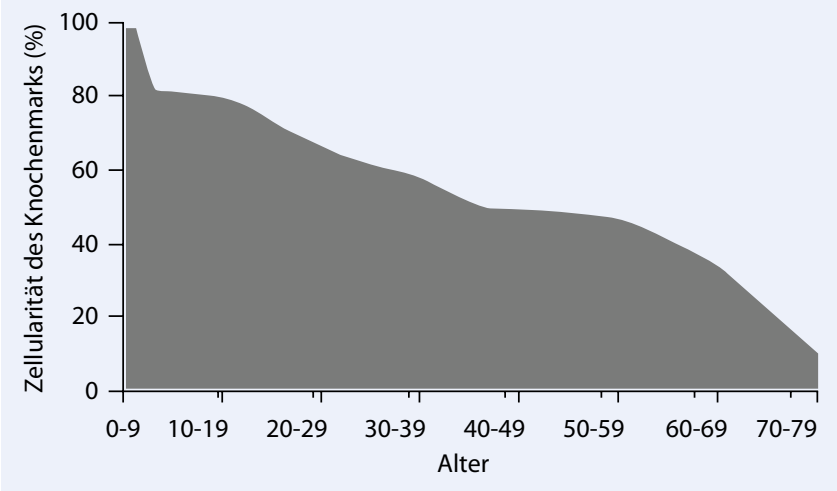




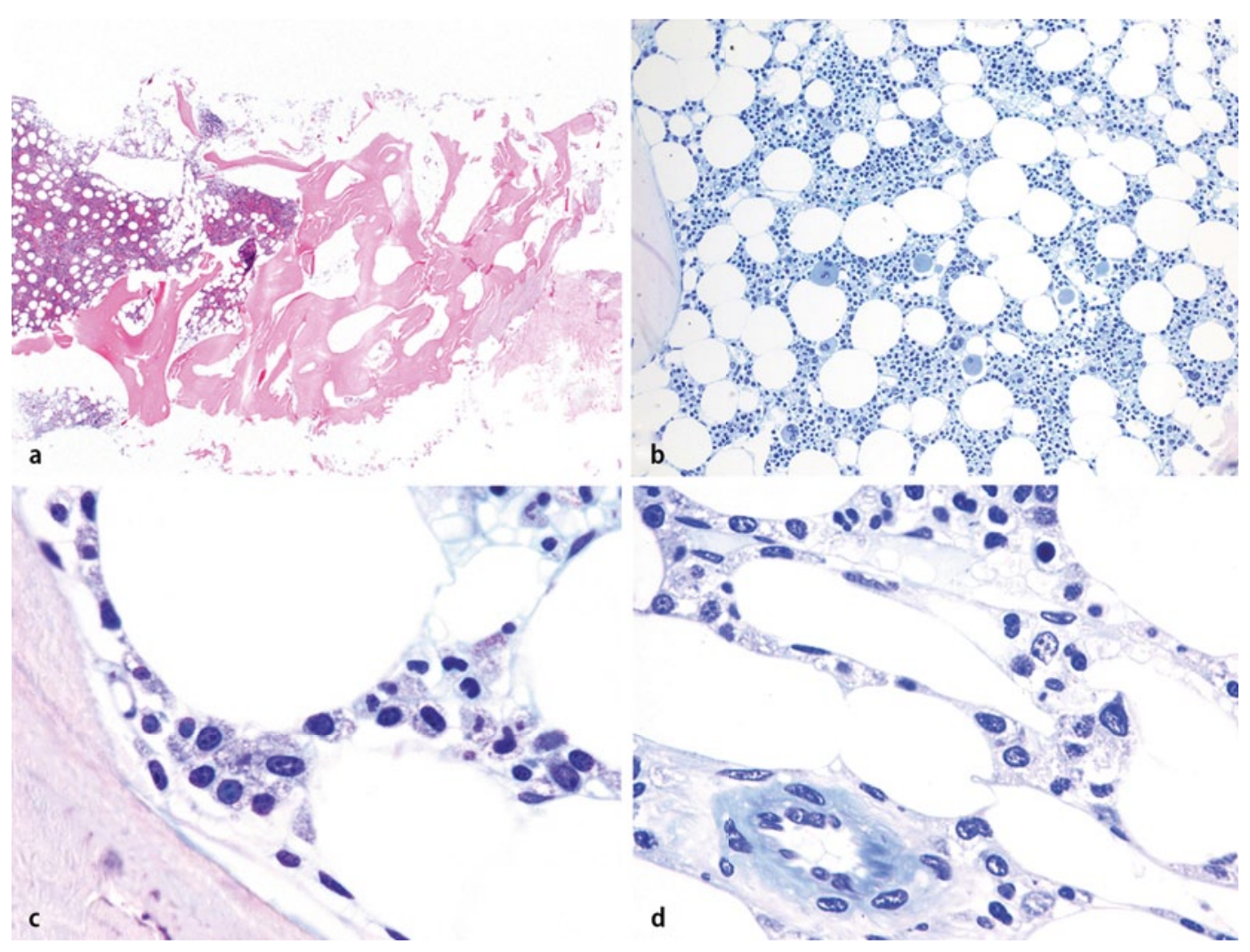

Abb. $2<$ a Ausgeprägter subkortikaler Fettgürtel (Hämatoxylin-Eosin-Färbung, Vergr. 25-fach). b Zellverteilung im normalen Knochenmark (MayGrünwald-Giemsa-Färbung, Vergr. 100-fach). c, d Physiologische Lokalisation der myeloischen Vorstufen peritrabekulär (c) und perivaskulär (d; MayGrünwald-Giemsa-Färbung, Vergr. 600-fach)

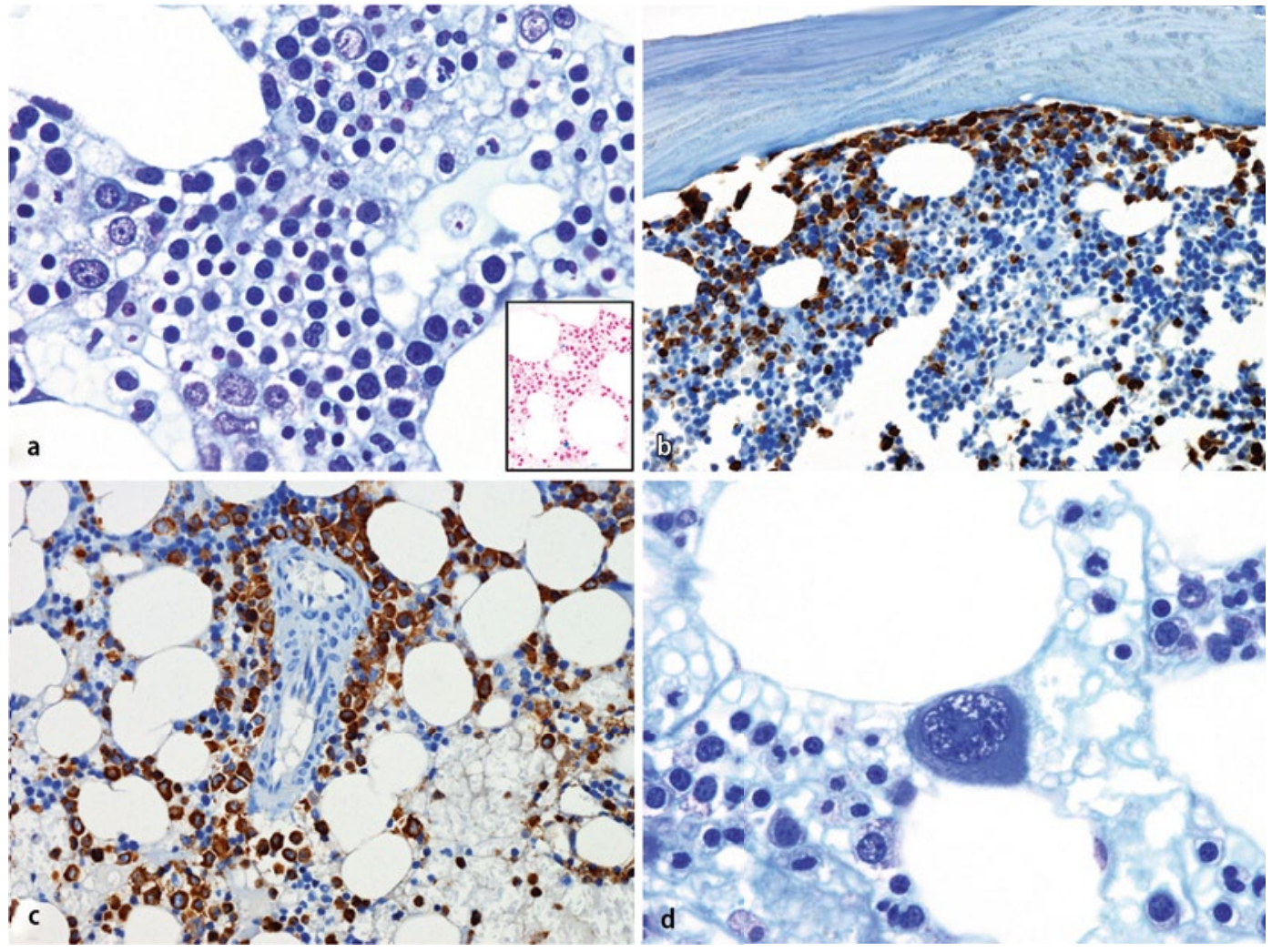

Abb. $3<$ a Erythron in Bildmitte (May-GrünwaldGiemsa-Färbung, Vergr. 400-fach), Inset: Eisengranula inmitten erythropoietischer Zellgruppe (Berlinerblau, Vergr. 200-fach). b, c Immunohistochemische Darstellung der myeloischen Zellen peritrabekulär (b) und perivaskulär (c Myeloperoxidase,

b Vergr. 200-fach, c Vergr. 400-fach). d Sinusnaher lokalisierter Megakaryozyt (May-Grünwald-GiemsaFärbung, Vergr. 400-fach) 


\section{Markraumarchitektur}

Die normale Markarchitektur zeigt die Kompartimente der Hämatopoiese zwischen den Spongiosabälkchen (- Abb. 2b).

Myeloische Vorstufen liegen normalerweise peritrabekulär und -vaskulär (- Abb. 2c, d), reife granulopoietische Formen sind markzentral nachweisbar. Megakaryozyten und erythropoietische Zellnester finden sich markzentral, diffus verteilt, bevorzugt perisinusoidal [1, 12].

\section{Zelluläre Elemente im Mark}

Basis der Hämatopoiese bilden die hämatopoietischen Stammzellen, die man in der Biopsie morphologisch nicht erkennen kann.

\section{Erythropoiese}

Kernhaltige Vorstufen der Erythropoiese liegen in Form von markzentralen Zellgruppen, sog. Erythronen, vor (• Abb. 3). In unmittelbarer Nachbarschaft, gelegentlich auch im Zentrum dieser Gruppen, liegt ein Makrophage („Mutterzelle"), der defekte erythropoietische Zellen oder Kernbruchstücke aufnimmt. Häufig ist mittels Eisenfärbung Hämosiderin im Zytoplasma nachweisbar (• Abb. 3a, Inset).

Die erythropoietischen Zellnester enthalten das Reifungsspektrum vom Erythroblasten bis zum Normoblasten. Frühe Erythroblasten, die Transferrin und Glykophorin exprimieren, sind große Zellen mit rundlichen Kernen, diffus verteiltem Chromatin und multiplen kleinen Nukleolen. Reife schmalzytoplasmatische Zellen zeigen ein dicht kondensiertes Chromatin in runden Zellkernen. Je reifer die erythropoietischen Zellen werden, desto schwächer wird die Expression von Transferrin, während die Glykophorinexpression gleich intensiv bleibt [5]. Der Reifungsgrad der erythropoietischen Zellen setzt sich aus Kerngröße, Chromatingehalt, Nukleolenanzahl und Basophilie des Zytoplasmas zusammen. Erythropoietische Zellnester liegen bevorzugt in der Nähe von Sinusoiden. Das Mengenverhältnis Myelopoiese/Erythropoiese liegt zwischen 1,5:1-3:1.

Pathologe 2012 - 33: 496-507 DOI 10.1007/s00292-012-1649-x

(c) Springer-Verlag Berlin Heidelberg 2012

\section{A. Tzankov · S. Dirnhofer · C. Beham-Schmid Normales Knochenmark und häufige reaktive Veränderungen}

\section{Zusammenfassung}

Die histologische

Knochenmarkuntersuchung spielt, wegen ihrer großen Aussagekraft bei relativ geringem Aufwand eine bedeutende Rolle in der Diagnostik von hämatologischen und nichthämatologischen Erkrankungen. Dafür ist die Kenntnis des normalen Knochenmarks mit seiner individuellen, insbesondere altersabhängigen, Variabilität, unentbehrlich. Neben entnahmebedingten Artefakten, die falsch interpretiert werden können, oder suboptimal fixierten bzw. verarbeiteten Biopsien, die diagnostisch überbewertet werden, gibt es eine Vielfalt von reaktiven Knochenmarkveränderungen, die einen neoplastischen Prozess vortäuschen und zu schwerwiegenden diagnostischen Fehlern führen können. Bei derartigen nichtneoplastischen Veränderungen können ein oder auch mehrere Kompartimente der Hämatopoiese qualitativ und quantitativ betroffen sein. Es kann zu Verteilungs- bzw. Architekturstörungen und/oder zu Veränderungen des Knochenmarkstromas kommen. Eine optimale Knochenmarkdiagnostik erfordert, neben Spezialfärbungen, zusätzlich oftmals immunhistochemische Untersuchungen und manchmal molekularpathologische Analysen. Mehr als bei anderen Organbiopsien ist die Kenntnis klinischer Befunde, insbesondere vorangegangener Therapien, relevant, um eine korrekte Diagnose zu stellen. In diesem Beitrag sind neben dem normalen Knochenmark die häufigsten reaktiven Veränderungen dargestellt.

Schlüsselwörter

Knochenmark · Reaktive Veränderungen ·

Färbung $\cdot$ Immunhistochemie .

Molekularpathologische Analysen

\section{Normal bone marrow and common reactive alterations}

\section{Abstract}

Histological examination of bone marrow biopsies is an important and powerful diagnostic tool to assess various hematological and non-hematological disorders. Morphological examination of such biopsies requires knowledge of the composition of normal bone marrow and its variations, such as agerelated changes. Diagnostic problems may arise due to poor specimen quality, insufficient sections or stainings and insufficient experience with reactive bone marrow changes which occasionally resemble neoplastic disorders. Reactive bone marrow processes can affect one or more hematopoietic cell lines, lead to disruption of the normal architecture and specifically affect the bone marrow stro- ma. Optimal bone marrow diagnosis requires adequately stained slides and, when needed, immunophenotyping and molecular examinations. Furthermore, rather than biopsy interpretation of other organs, pathologists routinely need clinical history information for correct interpretation and diagnosis of bone marrow changes. In this article, the normal features of bone marrow as well as the most frequent reactive bone marrow alterations are described.

\section{Keywords}

Bone marrow - Reactive Alterations . Staining $\cdot$ Immunophenotyping $\cdot$ Molecular examinations
Hilfreich zur Erkennung erythropoietischer Vorstufen und zur Unterscheidung von myeloischen Zellen ist die Detektion rundkerniger Zellen in sinusnahen Gruppen (Erythrone; [28]). Proerythroblasten und Normoblasten sind mitotisch sehr aktiv und weisen die höchste Poliferationsfraktion auf.

Gelegentlich sind Normoblasten aufgrund von Schrumpfungsartefakten nach Entkalkung schwer von lymphatischen Zellen zu unterscheiden. Besonderes Augenmerk muss in solchen Fällen auf die
Chromatinverteilung im Zellkern gelegt werden, die bei den Normoblasten gleichmäßig kondensiert scheint und in Lymphozyten gröbere Verklumpungen erkennen lässt.

\section{Granulopoiese}

Im normalen Knochenmark ist die Granulopoiese mit 50-70\% der kernhaltigen Zellen die quantitativ dominierende Zellreihe. Die früheste am histologischen Schnitt erkennbare granulopoietische Vorläuferzelle ist der Myeloblast, der sich 
meist unmittelbar an der Bälkchenoberfläche oder periarteriolär erkennen lässt. In größerer Anzahl zeigen sich Promyelozyten und Myelozyten. Diese liegen ebenfalls in der Generationszone der Granulopoiese peritrabekulär und periarteriell (- Abb. 2c, d). Mit zunehmender Reifung findet man granulopoietische Zellen abseits der Trabekel und Gefäße. Mit einem Antikörper gegen Myeloperoxidase (stark positiv in den Promyelozyten, etwas schwächer positiv in späteren Entwicklungsstufen) und/oder CD15 (positiv in Metamyelozyten und stark positiv in Granulozyten) kann diese Zellverteilung dargestellt werden (• Abb. 3b, c; [1]). Die Granulozyten überschreiten schließlich die Gefäßwand, um in den Blutkreislauf überzutreten.

Neutrophile und eosinophile Granulozyten sind leicht unterscheid- und identifizierbar, selten jedoch kann man basophil differenzierte Formen mit konventionellen Färbungen erkennen. Hierzu werden (immun-)histochemische Färbungen benötigt (z. B. May-Grünwald-Giemsa-Färbung, Tryptase).

\section{Monozytopoiese}

Im normalen Knochenmark sind Monozyten schwer erkennbar. Die in geringer Anzahl vorhandener Zellen liegen meist inmitten hämatopoietischer Zellnester oder periarteriell. Sie sind etwas größer als Granulozyten, zeigen lobulierte Kerne und ähneln somit den plumpen stabkernigen Formen. Immunhistochemisch sind monozytär differenzierte Zellen mit CD11c, CD14, CD68, CD163 oder Lysozym darstellbar [30].

Im Knochenmark (und anderen Geweben) reifen Monozyten zu Makrophagen mit komplexen, verzweigten $\mathrm{Zy}$ toplasmata, die in großer Anzahl diffus verteilt im Knochenmark zu finden sind. Diese Makrophagen sind ebenfalls CD68 positiv und können praktisch als interne Positivkontrolle dieser Färbung dazu gezogen werden. Neben Hämosiderin können diese Zellen andere Substanzen, wie beispielsweise Lipid, hämatopoietische Zellen oder Kerntrümmer speichern.

\section{Megakaryopoiese}

Megakaryozyten sind mit einem Durchmesser von 12-150 $\mu \mathrm{m}$ die größten kern- haltigen Zellen im normalen Knochenmark und liegen bevorzugt perisinusoidal (- Abb. 3d). Da Sinusoide oft nicht erkennbar sind, scheinen Megakaryozyten meist diffus verteilt in den Markräumen zu liegen. Megakaryozyten finden sich entweder isoliert oder in lockeren Gruppen bis zu maximal 5 Zellen. Sie zeigen eine unterschiedlich ausgeprägte Zytoplasmagranulierung mit helleren und dunkleren Arealen, der Kern ist unterschiedlich stark gelappt. Häufig, v. a. wenn Megakaryozyten vermehrt sind, sieht man eine Emperipolesis [5], die keine pathologische Bedeutung hat.

Unterschieden werden 3 Reifungsstadien:

1. Megakaryoblast mit ovalem bis nierenförmigen Kern, basophilem Zytoplasma und einem Durchmesser bis $20 \mu \mathrm{m}$;

2. Promegakaryozyt mit weniger basophilem Zytoplasma aber häufiger Granulierung und einem Durchmesser von etwa $20-80 \mu \mathrm{m}$;

3. reifer Megakaryozyt mit Eosinophilie des Zytoplasmas und unterschiedlicher Granulierung sowie einem Durchmesser bis $150 \mu \mathrm{m}$.

Die durchschnittliche Anzahl von Megakaryozyten am hämatoxylin- und eosingefärbten Schnittpräparat ist $8-15 / \mathrm{mm}^{2}$, das entspricht zwischen 2 und 5 Megakaryozyten/HPF. Immunhistochemisch (Faktor VIII, CD42b oder CD61) lassen sich mehr (bis zu $25 / \mathrm{mm}^{2}$ ), v. a. kleine Vorstufen, identifizieren [12].

\section{Lymphozyten}

Im normalen Knochenmark zeigen sich Lymphozyten interstitiell, gelegentlich in kleinen Aggregaten oder auch in Form von nodulären Infiltraten (s. unten). Während im kindlichen Knochenmark Lymphozyten 30-60\% der kernhaltigen Zellen ausmachen können, sind beim Erwachsenen $10-20 \%$ nachweisbar, wobei mehr T(CD3) als B-Zellen (CD20) vorhanden sind. Das Verhältnis von T:B reicht von $4: 1$ bis $6: 1[36]$.

\section{Plasmazellen}

Plasmazellen sind in geringer Anzahl (weniger als 5\% der kernhaltigen Zellen) in jedem Knochenmark zu finden [12].

\section{Hier steht eine Anzeige.}



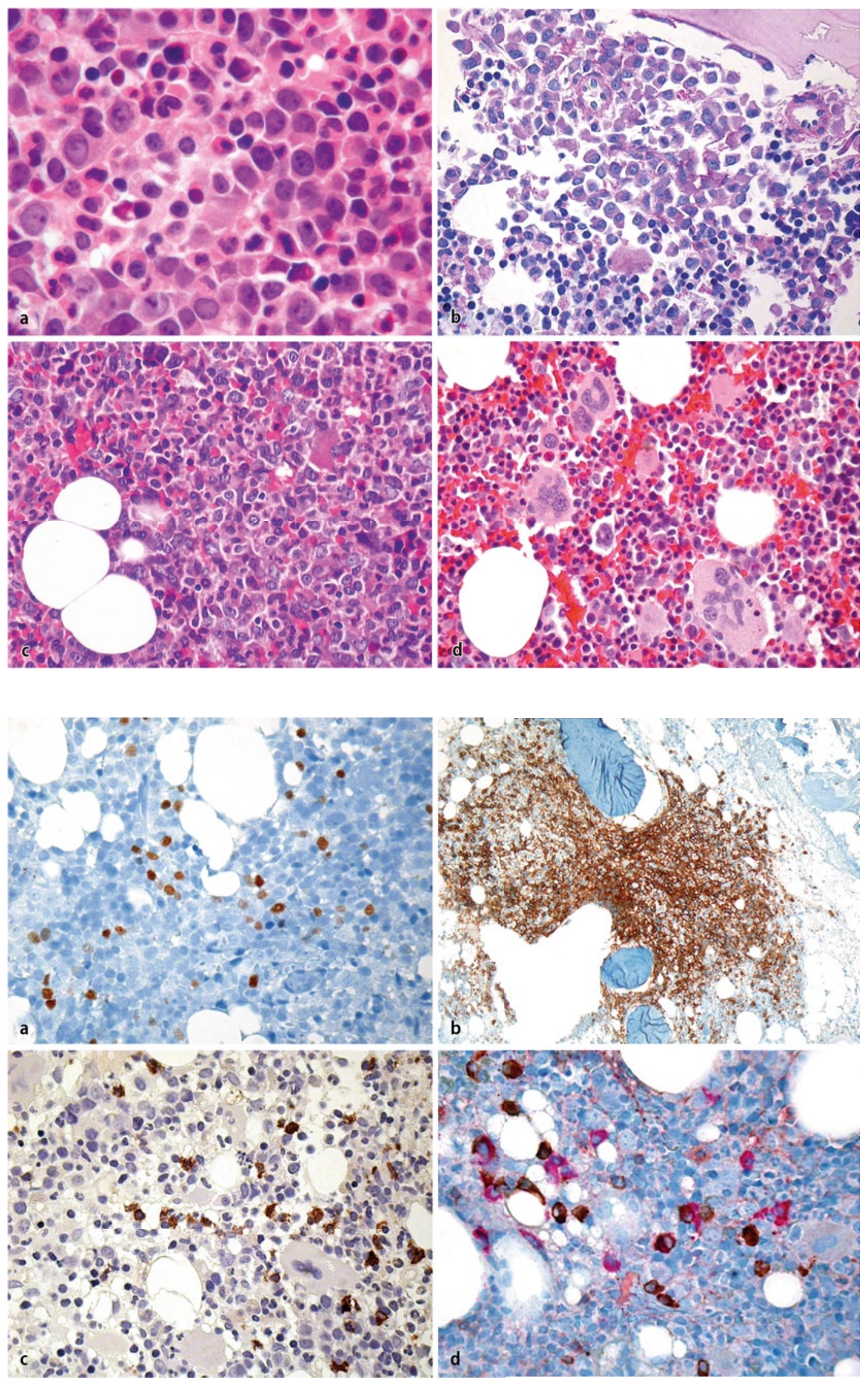

Abb. $4<$ a Megaloblastäre Veränderungen der Erythropoiese und Segmentierungsstörungen der Myelopoiese bei VitaminB12-Mangel (Hämatoxylin-Eosin-Färbung, Vergr. 400-fach). b Reifungsstopp der Myelopoiese auf Ebene der Promyelozyten nach Carbamazepingabe (Perjodschiffsäure-Färbung, Vergr. 200-fach). c Massive myelopoietische Hyperplasie mit Linksverschiebung nach Gabe von "granulocyte colony-stimulating factor" (Hämatoxylin-Eosin-Färbung, Vergr. 200-fach). d Myeloproliferative Veränderungen des Knochenmarks nach Erythropoietingabe (Hämatoxylin-Eosin-Färbung, Vergr. 120-fach)
Abb. $5<$ a Vermehrung von Hämatogonen nach Chemotherapie (TdT, Vergr. 200-fach). b Adhäsivität eines B-Zelllymphomaggregats zu den Knochentrabekeln (CD20, Vergr. 100-fach). c Interstitielle Vermehrung von großen, granulären T-Zelläquivalenten im Knochenmark nach allogener hämatopoietischer Stammzelltransplantation (CD57, Vergr. 200-fach). d Polyklonale Plasamazytose, dargestellt in der Doppelfärbung (braun kappa, rot lambda, Vergr. 320-fach) 
Meist liegen sie um kleine Blutgefäße herum, können aber auch diffus verteilt einzeln in den Markräumen auftreten.

\section{Mastzellen}

Physiologisch finden sich Mastzellen in geringer Anzahl diffus verteilt im Interstitium, bevorzugt perivaskulär sowie in der Peripherie reaktiver Lymphozytenaggregate. Um diese Zellen nicht zu übersehen, werden metachromatische Färbungen (z. B. May-Grünwald-Giemsa-Färbung) und immunhistochemische Untersuchungen (z. B. Tryptase und CD117) benötigt [37].

\section{Knochenmarkstroma}

Die Hämatopoiese ist eingebettet in einem bindegewebigen Stroma bestehend aus Fettzellen, Blutgefäßen, Nerven, Fibroblasten und spärlich Retikulinfasern. Die dünnwandigen Sinusoide, durch die die neugebildete Hämatopoiese in den Kreislauf gelangt, sind am histologischen Schnittpräparat häufig kollabiert und schwer erkennbar. Die im normalen Mark vorhandenen zarten Retikulinfasern zeigen sich bei Spezialfärbungen perivaskulär und peritrabekulär [1]. Hämosiderinspeichernde Zellen sind mittels Spezialfärbung unregelmäßig verteilt zu finden. Die Quantität eisenspeichernder Zellen kann graduiert werden [13].

\section{Häufige reaktive Veränderungen der Hämatopoiese}

\section{Knochenmarkhypoplasie/-aplasie}

Die Feststellung einer Knochenmarkhypoplasie bzw. -aplasie muss, wie erwähnt, zum pseudoaplastischen subkortikalen Markraumkompartiment abgegrenzt werden. Sollte es sich hierbei nicht um dieses Kompartiment handeln, so ist die Feststellung eines hypoplastischen bzw. aplastischen Knochenmarks weiter interpretationsbedürftig, zumal es sich um Ausdruck angeborener oder erworbener Erkrankungen z. B. aus dem Formenkreis der aplastischen Anämien handeln kann $[9,12]$.

Die Feststellung einer Hypozellularität ist neben der Zytopenie eines von zwei erforderlichen Kriterien für die Diagno- se einer aplastischen Anämie [24] und darf nur an repräsentativen Biopsien vorgenommen werden. Typischer Befund in diesem Zusammenhang ist die hochgradige Verminderung bzw. das vollkommene Fehlen der Myelopoiese bei gleichzeitig fokal aufrecht erhaltenen erythropoietischen Inseln. Im Stroma zeigen sich Plasmazellen, Mastzellen und Makrophagen sowie gelegentlich lymphoide Aggregate [12].

Da aplastische Anämien auch paraneoplastisch im Rahmen von lymphoproliferativen Prozessen auftreten können [26], sollten solche lymphoiden Infiltrate zur Dignitätsbeurteilung weiter immunophänotypisch untersucht werden. Die Differenzialdiagnose zu hypoplastischen myelodysplastischen Syndromen erfordert nicht nur die akkurate Untersuchung der verbleibenden Megakaryozyten auf allfällige Dysplasiezeichen, sondern auch die Quantifizierung der Blasten mittels CD34-Immunohistochemie [24]. Selten können sich auch akute lymphoblastische Leukämien (ALL) mit einem hypoplastischen Knochenmarkbild präsentieren, sodass hier entsprechende immunohistochemische Untersuchungen ebenfalls erforderlich sein können [24].

\section{Veränderungen der roten Zellreihe}

Eine Verminderung der roten Zellreihe kann angeborener (Diamond-BlackfanSyndrom) oder erworbener Natur sein [9]. Die häufigsten erworbenen Aplasien der roten Zellreihe sind mit einer Parvovirus-B19-Infektion assoziiert (transient), idiopathisch (autoimmun) oder treten im Rahmen von allogenen hämatopoietischen Stammzelltransplantationen über die AB0-Barriere auf. Lediglich die Parvovirus-B19-Infektion zeigt pathognomonische Befunde in Form sog. Lampionzellen [21]. Es handelt sich um vergrößerte erythroide Vorläuferzellen mit zyoplasmatischer Vakuolisierung und intranukleären Einschlüssen mit peripher kondensiertem Chromatin. Entsprechende immunohistochemische Untersuchungen können den Nachweis von Virusinklusionen in diesen Zellen erbringen. Eine wichtige Erkrankung, die überdurchschnittlich häufig mit einer reinen Aplasie der roten Zellreihe assoziiert ist, stellt die Leukämie

\section{Hier steht eine Anzeige.}




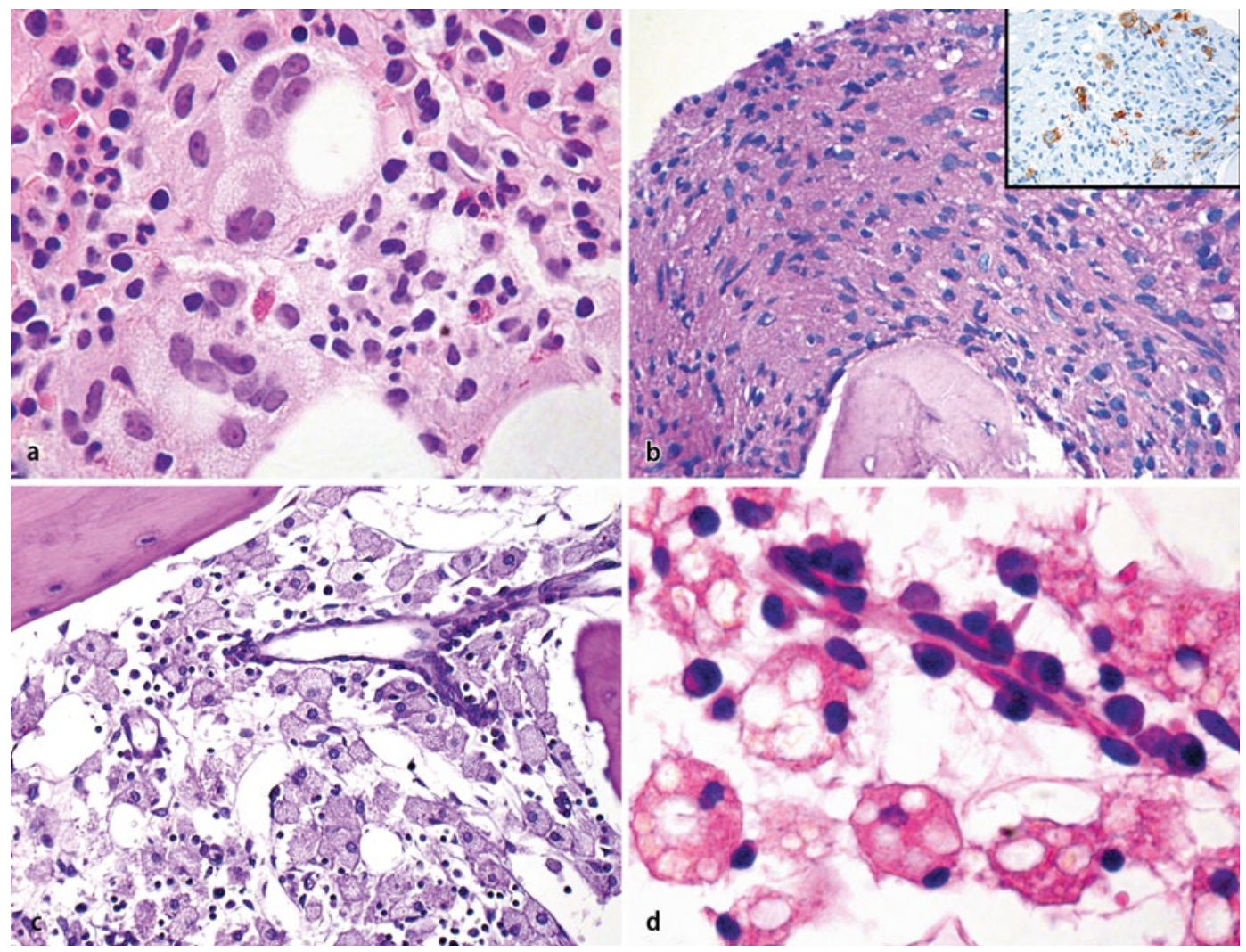

Abb. $6<$ a Kleines Ringgranulom bei Zytomegaloviruserstinfektion (Hämatoxylin-Eosin-Färbung, Vergr. 400-fach). b Totale Markverdrängung durch Granulome im Rahmen eines disseminierten HodgkinLymphom-Befalls [Perjodschiffsäure (PAS)- Färbung, Vergr. 200-fach]. Inset: Fokus mit Hodgkinund Reed-Sternberg-Zellen (CD30, Vergr. 150-fach). c Diffuse, PAS-negative Schaumzellvermehrung nach myeloablativer Therapie wegen eosinophiler Leukämie (PAS, Vergr. 150-fach). d Hämophagozytierende Histiozyten und Plasmazytose bei EpsteinBarr-Virus-getriggertem, hämophagozytischem Syndrom (Hämatoxylin-EosinFärbung, Vergr. 400-fach)
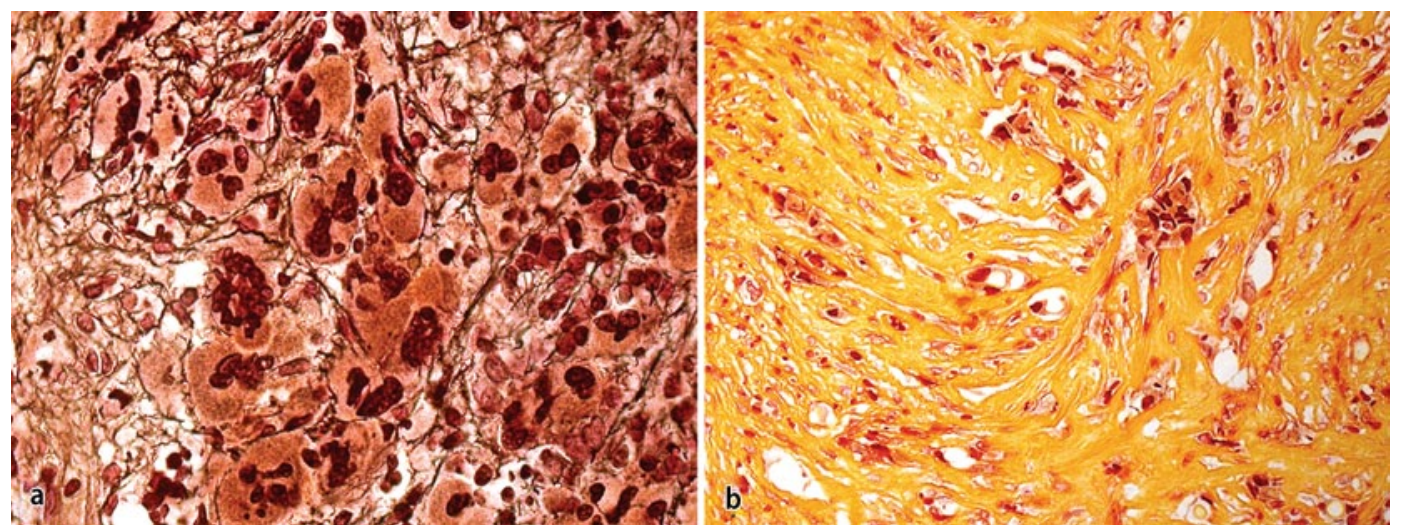

Abb. $7<$ a Diffuse Retikulinfaserfibrose Grad 3 nach Thiele bei myeloproliferativer Neoplasie (Gömöri-Färbung, Vergr. 320-fach). b Diffuse Kollagenfaserfibrose bei metastasiertem Magenkarzinom (GömoriFärbung, Vergr. 200-fach)

großer granulärer T-Zellen dar (T-LGL), weshalb bei Feststellung solcher Veränderungen proaktiv auch an eine allfällig okkulte T-LGL im Knochenmark gedacht werden muss [33].

Hyperplasie, Linksverschiebung und megalo- bzw. makroblastäre Veränderungen der Erythropoiese sowie Kerndefekte der Myelopoiese ( $\bullet$ Abb. 4a) sind typische Zeichen eines Vitamin-B12- bzw. Folsäuremangels [12]. Die Zahl der Erythroblasten kann in solchen Situationen sehr hoch werden, sodass die Unterscheidung zu einer Erythroleukämie sich schwierig gestaltet. Nur die integrative Interpreta- tion unter Berücksichtigung der peripheren Blutwerte und der Anamnese führt zur richtigen Diagnose. Ein gesteigerter peripherer Erythrozytenumsatz, z. B. im Rahmen autoimmuner, hämolytischer Anämien, wird oft von einer Vergrößerung der Erythrone begleitet.

\section{Veränderungen der Myelopoiese}

Praktisch jede eitrige Entzündung wird von einer myelopoietischen Hyperplasie mit Linksverschiebung begleitet, im Extremfall bis hin zur leukämoiden Reaktion. Septische Zustandsbilder führen über den
Mechanismus des gesteigerten peripheren Plättchenverbrauchs auch zu einer megakaryopoietischen Hyperplasie und Linksverschiebung. Ferner können Steroidgaben und Expositionen gegenüber hämatopoietischen Wachstumsfaktoren [16] $\mathrm{zu}$ ähnlichen Veränderungen führen (- Abb.4b).

Segmentierungsstörungen/Überreife der Myelopoiese kann Ausdruck eines Vitamin-B12-Mangels sein [12]. Verminderungen, Reifungsstopp bis hin zur Aplasie der Myelopoiese werden in Rahmen von toxischen bzw. immunoallergischen Medikamentenreaktionen (z. B. 
Clozapin, Metamizol oder Thiamazol; [18]), aplastischen Anämien, bei anderen Autoimmunerkrankungen [6] oder bei Zytomegalovirusprimärinfektionen [20] beobachtet ( $\bullet$ Abb. 4c). Die besondere Empfindlichkeit der Myelopoiese gegenüber Medikamenten, speziell Arylamine, wird mit der aktiven intrazellulären Metabolisierung durch die Myeloperoxidase und der In-situ-Generation von freien Radikalen erklärt [23]. Paraneoplastische Neutropenien bei T-LGL sind oft von einer deutlichen Linksverschiebung der Myelopoiese begleitet [12].

\section{Veränderung der Megakaryozyten}

Das klassische Beispiel einer Vermehrung normaler oder leicht linksverschobener Megakaryozyten im Knochenmark stellt die Immunthrombozytopenie (ITP) dar [35]. Die ITP ist eine erworbene Erkrankung, bei der Autoantikörper gegen Oberflächenantigene der Plättchen ihre Halbwertzeit verringern [25]. Eine Differenzialdiagnose stellt die Thrombozytopenie bei HIV-infizierten Individuen dar, bei denen es ebenfalls zu einer Vermehrung kleiner Megakaryozyten mit pyknotischen Kernen kommen kann.

Erythropoietin und Thrombopoietin können transiente morphologische Veränderungen induzieren, die an myeloproliferative Neoplasien erinnern (- Abb. 4d; [12]).

Eine Verminderung der Megakaryopoiese ist meistens parainfektiöser oder medikamentös-toxischer Natur, gelegentlich ein Begleitphänomen von T-LGL [11]. Medikamente wie Mykophenolat, Valproinsäure und Tacrolimus können ebenfalls selektiv die Megakaryopoiese verändern [2]. Über angeborene Megakaryozytenerkrankungen wird in dieser Übersichtsarbeit nicht berichtet. Diesbezüglich wird auf die einschlägige Literatur verwiesen [12].

\section{Lymphozytosen}

\section{Hämatogonen}

Hämatogonen sind gutartige lymphoide Vorläuferzellen mit morphologischen und phänotypischen Merkmalen, welche Überlappungen mit den ALL-Blasten [31]. Sie sind besonders zahlreich in pä- diatrischen Knochenmarkbiopsien, speziell nach Infekten und insbesondere nach Chemotherapie [31]. Hämatogonen sind kleine bis mittelgroße lymphoide Formen mit wenig Zytoplasma und dichtem, homogenen Chromatin ohne Ausbildung von Nukleolen (• Abb.5a). Sie exprimieren heterogen CD34, TdT, CD20 und CD79a sowie schwach CD10. Im Gegenteil zu den ALL-Blasten zeigen die Hämatogonen eine diskordante Expression [17] zwischen CD34/TdT und CD123 sowie zwischen CD34/TdT und LMO2. Meistens sind Hämatogonen interstitiell verteilt. Sehr selten bilden sie perivaskuläre Aggregate aus [12]. Cluster von mehr als 5 TdT oder CD34-positive Blasten werden bei Patienten mit vorangegangener ALL als verdächtig für ein Rezidiv angesehen [31].

\section{Lymphoide Aggregate}

Reifzellige lymphoide Aggregate im Knochenmark werden in knapp der Hälfte der Knochenmarkbiopsien älterer Individuen festgestellt [14]. Die Identifikation nodulärer lymphoider Aggregate bei jüngeren Patienten bedarf einer klinisch-pathologischen Korrelation, da sie überdurchschnittlich häufig bei Kollagenosen sowie entzündlichen und infektiösen Erkrankungen vorkommen [32].

Die gutartigen lymphoiden Aggregate im Knochenmark sind meist perivaskulär lokalisiert, gut abgrenzbar und können gelegentlich Keimzentrumsstrukturen oder zentrale granulomatöse Ansammlungen aufweisen. Diese Aggregate zeigen einen zonalen Aufbau mit einer T-Zell-prädominanten Peripherie und einem B-Zell-prädominanten Zentrum. Bei Gegenüberstellung der Färbungen für CD20 und CD5 zeigt sich keine Expression von CD5 auf B-Zellen [36].

Die wichtigsten Differenzialdiagnosen sind reifzellige B- oder T-Zell-Neoplasien. Erfolgen muss die die Abgrenzung integrativ unter Berücksichtigung

- der Lokalisation: Adhäsivität der lymphoiden Aggregate zu den Knochentrabekel ist ein Malignitätszeichen (- Abb. 5b);

- der Größe: Reaktive lymphoide Aggregate bei nicht immunsupprimierten Individuen sind selten größer als $200 \mu \mathrm{m})$;

\section{Hier steht eine Anzeige.}




\section{Infobox 1: Interpretationshilfe zur Beurteilung und Diagnostik von Knochenmarkbiopsien}

1. Länge und Beurteilbarkeit

2. Knöcherne Strukturen
a) Regelrecht
b) Pathologisch
I. Osteopenie
II. Knochenumbau
III. Osteosklerose
IV. Renale Osteodystrophie
V. Osteomalazie

3. Zellularität (unter Berücksichtigung des Patientenalters und Angabe des Prozentwertes)
a) Hypozellularität
I. Subkortikale Pseudoaplasie
II. Aplastische myelodysplastische Syn- drome/akute myeloische Leukämie/ akute lymphatische Leukämie/Haar- zellleukämie
III. Aplastische Anämie
b) Hyperzellularität
I. Leukämoide Reaktion
II. Regeneration
III. Applikation von Wachstumsfaktoren
IV. Neoplasie

4. Veränderungen der Erythropoiese
a) Hypoplasie
I. Angeboren (z. B. Diamond-Blackfan- Syndrom)
II. Parvovirus-B19-Infektion
III. Autoimmunität IV. T-LGL (Große granuläre-T-Zellen)-
Leukämie
b) Hyperplasie
I. (Autoimmune) hämolytische Anämie
II. Vitamin-B12- und/oder Folsäure- mangel
III. Applikation von Wachstumsfaktoren IV. Neoplasie

5. Veränderungen der Myelopoiese
a) Hypoplasie
I. Medikamentennebenwirkung
II. Aplastische Anämie
III. T-LGL-Leukämie
b) Hyperplasie
I. Leukämoide Reaktion
II. Applikation von Wachstumsfaktoren
III. Neoplasie

6. Veränderungen der Megakaryopoiese
a) Hypoplasie
I. Angeboren
II. Medikamentennebenwirkung
III. T-LGL-Leukämie

\section{Infobox 1: (Fortsetzung)}

b) Hyperplasie

I. Gesteigerter Plättchenumsatz

(z. B. Immunthrombopenie)

II. Neoplasie

7. Lymphozytose

a) Aggregate

I. Reaktiv/unspezifisch

II. Autoimmunität

III. Neoplasie

b) Hämatogonen

I. Therapienebenwirkung

II. Akute lymphatische Leukämie

c) T-LGL

I. Immunologisch/parainfektiös

II. T-LGL-Leukämie

d) Plasmazytose

I. Reaktiv

II. Neoplastisch

8. Histiozytose

a) Granulomatös

I. Infektiös

II. Paraneoplastisch

III. Therapienebenwirkung

IV. Idiopathisch

b) Diffus

I. Abräumreaktion

II. Speicherkrankheiten

III. Infektiös

IV. Hämophagozytose

V. Neoplasie

9. Fibrose
a) Neoplasie inklusive Metastasen
b) Autoimmun
c) Metabolisch
d) Therapienebenwirkung

- der Zellzusammensetzung: Ausbildung von echten Keimzentren ist mit Ausnahme von Marginalzonenlymphomen und multizentrischer Castleman-Erkrankung meist Zeichen eines reaktiven Geschehens sowie

mit dem Einsatz immunphänotypischer Untersuchungen:

- Expression von Cyclin D1 und SOX11 beim Mantelzelllymphom,

- Nachweis von BCL2-Rearrangement mittels Fluoreszenz-In-situ-Hybridisierung (FISH) beim follikulären Lymphom $[12,36]$.

PCR-basierte Klonalitätsuntersuchungen müssen stets mit Vorsicht interpre- tiert werden, da sowohl falsch-positive, als auch falsch-negative Ergebnisse vorkommen können [10]. Bei schwer immunsupprimierten Patienten, insbesondere bei Patienten mit HIV, können lymphoide Aggregate eine deutliche Pleomorphie aufweisen [12, 29].

Die Abgrenzung zu immunsuppressionsassoziierten lymphoproliferativen Erkrankungen erfordert in solchen Fällen den Nachweis/Ausschluss einer Klonalität und Nachweis/Ausschluss sowie Mengenbestimmung allfällig Epstein-Barr-Virus (EBV)-infizierter Zellen.

\section{Proliferate großer granulärer T-Zellen}

Reaktive Expansionen von T-LGL wurden bei zahlreichen nichtneoplastischen $\mathrm{Zu}$ ständen festgestellt, wie z. B. virale Infektionen, speziell Zytomegalovirusinfektionen und Transplantationen $[1,2,38]$. Solche Expansionen sind meistens polyklonal, können allerdings auch einen dominanten T-Zell-Klon aufweisen (• Abb. 5c). Die Abgrenzung zu echten T-LGL-Leukämien muss integrativ unter Anwendung der von der Weltgesundheitsorganisation vorgesehenen Kriterien (Zytopenie, persistierende T-LGL-Lymphozytose, Klonalität der T-Zellen, Nachweis eines allfällig aberranten Immunphänotyps) erfolgen.

\section{Plasmazellproliferate}

Isolierte Plasmazellproliferate werden oft bei chronischen, entzündlichen oder infektiösen Prozessen sowie bei Patienten mit Autoimmunerkrankungen beobachtet. Solche Plasmazellproliferate sind meist perivaskulär und weisen in geringerem Maße zytologische Atypien auf.

Die Identifikation von Russell- und insbesondere von Dutcher-Körperchen sowie die nichtperivaskuläre Lokalisation der Plasmazellen lassen an die Möglichkeit einer neoplastischen Proliferation denken. Immunophänotypische bzw. In-situ-Analysen sind zur Klärung der definitiven Diagnose dann nötig. So sind neoplastische Plasmazellen für gewöhnlich leichtketten- bzw. schwerkettenrestringiert. Kappa/Lambda-Verhältnisse $>6: 1$ und Lambda/Kappa- >3:1 sind klonalitätsverdächtig, Kappa/Lamb- 


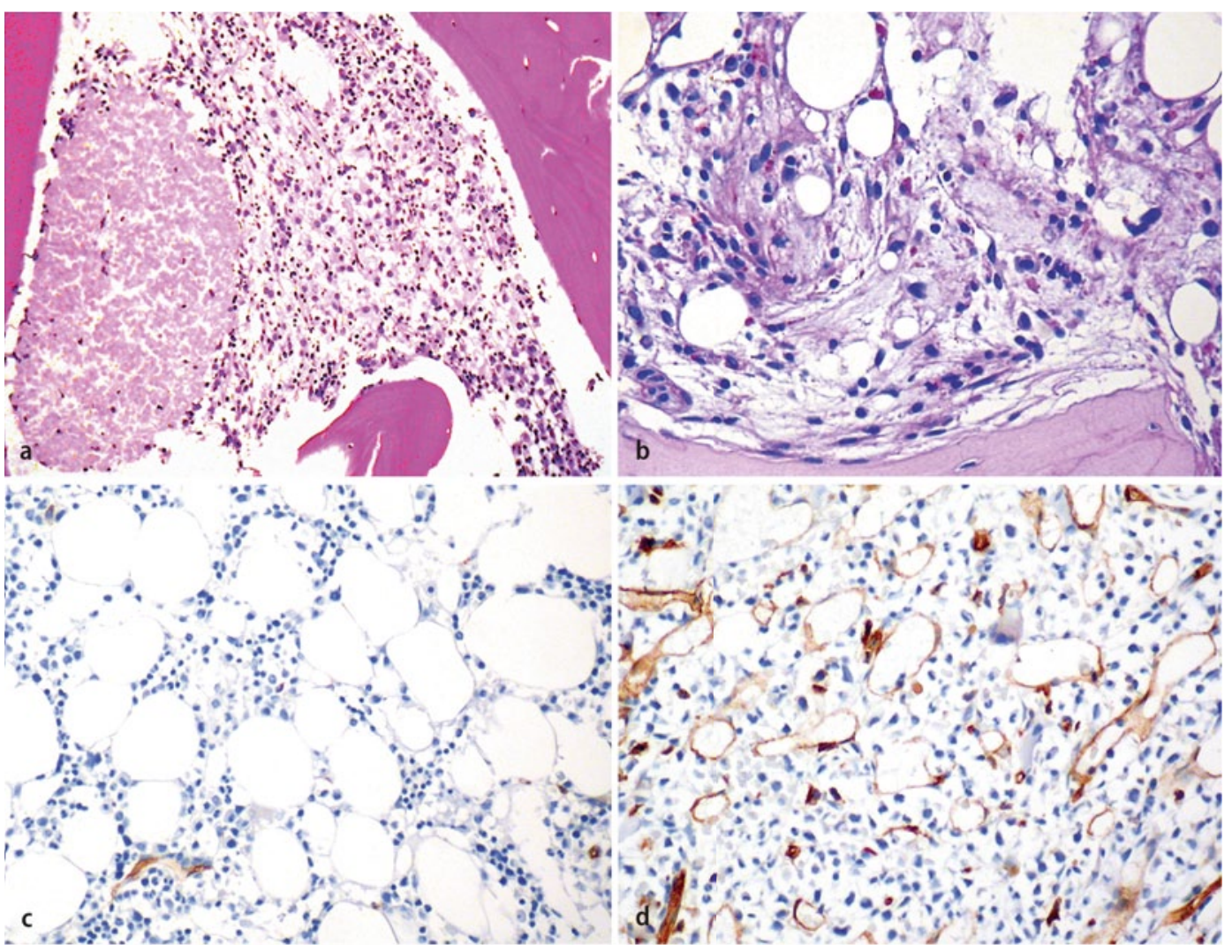

Abb. $8<$ a Fokale, zelluläre Tumornekrose im Prodromalstadium einer akuten monoblastären Leukämie mit MLL-Rearrangement. Der Patient war zum Zeitpunkt der Biopsieentnahme panzytopen, hatte jedoch keine nachweisbaren Blasten, retrospektiv sehr wohl MLL-Fusionstranskripte. Er entwickelte innerhalb eines Monats das Vollbild der Erkrankung (Hämatoxylin-Eosin-Färbung, Vergr. 64-fach). b Gelatinöse Transformation des Knochenmarkstromas nach myeloablativer Therapie wegen akuter myeloischer Leukämie (Perjodschiffsäurefärbung, Vergr. 250-fach). c Mikrogefäß im normalen Knochenmark (CD34, Vergr. 100-fach). d Vermehrte, dilatierte, pseudohämangiomatös-wirkende Mikrogefäße nach myeloablativer Therapie wegen akuter myeloischer Leukämie (CD34, Vergr. 150-fach)

da-Verhältnisse $>10: 1$, respektive Lambda/Kappa- $>5: 1$ klonalitätsbeweisend

(- Abb. 5d). Die normalen Verhältnisse von IgG:IgM:IgA:IgD betragen in etwa 5:1:0.5:0.1. Zudem zeigen neoplastische Plasmazellen oft eine Expression von CD56 (gewöhnlich negativ bei reaktiven Plasmazellproliferaten) und/oder Cyclin D1 (zu 40\% positiv in neoplastischen Plasmazellen; [3]).

Immunoblasten und Plasmablastenproliferate bei Patienten mit schweren systemischen Infektionen und bei Immunsupprimierten sind ein seltenes, aber gut dokumentiertes Phänomen [29]. Die Interpretation solcher Befunde kann nur integrativ unter Berücksichtigung der klinisch-pathologischen Besonderheiten dieser Patienten erfolgen, ggf. mithilfe von Zusatzuntersuchungen zum Nachweis/Ausschluss einer Klonalität sowie Nachweis/Ausschluss und Mengenbestimmung allfälliger EBV-infizierter Zellen.

\section{Granulome}

Verschiedene Typen von Knochenmarkgranulomen sind bekannt:
- verkäsende Granulome,

- epitheloidzellige nichtverkäsende Granulome,

- Lipogranulome,

- Ringgranulome und

- Fremdkörpergranulome.

Das Auftreten von Granulomen ist in etwa $10 \%$ der Knochenmarkbiopsien zu erwarten. Der exakte morphologische Typ des Granuloms ist wichtig, da es Rückschlüsse auf die Ätiologie erlaubt [12]. Zum Beispiel sind Ringgranulome überdurchschnittlich häufig mit Infektionen, wie z. B. Zytomegaloviruserstinfektion oder Q-Fieber assoziiert (• Abb. 6a). Andererseits treten Granulome häufig paraneoplastisch auf [7], sodass die Anfertigung weiterer Schnittstufen zum Ausschluss allfälliger Lymphominfiltrate, insbesondere Hodgkin-Lymphominfiltrate, ggf. mithilfe immunhistochemischer Untersuchungen, gerechtfertigt ist (- Abb.6b).

Die Interpretation der Granulome muss integrativ im Kontext der klinischen Präsentation, der (Reise- und Medikamenten-)Anamnese und der Serologie, sowie unter Einsatz von Spezialfär- bungen wie Ziehl-Neelsen, Perjodschiffsäure (PAS), Grocott, Mucicarmin, MayGrünwald-Giemsa u. a. erfolgen.

\section{Histiozytosen}

\section{Histiozytosen mit Hämophagozytose}

Die Identifikation hämophagozytierender Histiozyten im Knochenmark entspricht als isolierter Befund nicht der Diagnose eines hämophagozytischen Syndroms (- Abb. 6c). Diese Diagnose ist anhand klinischer Befunde und Laborwerte $\mathrm{zu}$ stellen [19].

Das Auftreten zahlreicher hämophagozytierender Histiozyten kann aber einerseits Ausdruck einer familiären hämophagozytischen Lymphohistiozytose (autosomal rezessive Erkrankungen mit Ausfall der „Natural-killer“- oder T-ZellFunktionen), andererseits Ausdruck sekundärer hämophagozytischer Syndrome, insbesondere infektgetriggert (z. B. EBV), sein [19]. Hilfreich zur Detektion hämophagozytierender Histiozyten sind einerseits die PAS-Färbung, andererseits die Färbung für CD68. Oft sind im Rahmen hämophagozytischer Syndrome auch an- 
dere Veränderungen, wie Plasmazytose, Fibrose oder Granulome, insbesondere Lipogranulome zu beobachten [12].

\section{Andere Histiozytosen}

Eine wichtige Form der diffusen Histiozytosen stellt die diffuse Vermehrung granulär-schaumig imponierender, PAS-positiver Histiozyten bei atypischer Mykobakteriose und bei Morbus Whipple dar [7]. Unter Berücksichtigung des klinisch-pathologischen Kontextes und mithilfe von Spezialfärbungen wie Ziehl-Neelsen oder Fite-Faraco kann eine richtige ätiologische Zuordnung erfolgen. Diffuse, schaumig imponierende, PAS-negative Histiozytosen können auch nach myeloablativer Therapie beobachtet werden ( $\bullet$ Abb. 6d).

Über Histiozytosen, die mit Speicherkrankheiten, z. B. Dyslipidämien, assoziiert sind, wird im vorliegenden Übersichtsartikel nicht berichtet. Diesbezüglich wird auf die einschlägige Fachliteratur verwiesen [12].

\section{Knochenmarkfibrose}

Basierend auf ihren Färbeeigenschaften werden im Knochenmark Retikulin von Kollagenfasern unterschieden. Retikulinfasern lassen sich mit Silberimprägnationstechniken nach Gömöri, GordonSweet und Novotny schwarz anfärben, während Kollagenfasern in diesen Färbungen pink-rötlich bzw. orange-gelblich imponieren ( $\mathbf{A b b}$. 7a, b). Diese Fasern werden von den Stromazellen des Knochenmarks produziert.

Eine Vermehrung der Retikulin- oder Kollagenfasern im Knochenmark ist stets ein Ergebnis einer Deregulation zwischen den faseraufbauenden und faserresorbierenden Prozessen [22]. Dem können sowohl neoplastische, als auch nichtneoplastische Erkrankungen zugrunde liegen. Nichtneoplastische Prozesse, die mit einer Knochenmarkfibrose assoziiert sind, sind z. B. die autoimmune Myelofibrose, Myelofibrosen bei Kollagenosen, HIV-assoziierte Myelopathien sowie metabolische Knochen- bzw. Knochemarkveränderungen, z. B. renale Osteopathie. Da zahlreiche neoplastische Prozesse ebenfalls mit Myelofibrose einhergehen, muss bei der Feststellung einer solchen eine neoplastische Ursache (z. B. myeloproliferative
Neoplasie, Haarzell-Leukämie, systemische Mastozytose, Lymphominfiltration, Metastasen) auf jeden Fall ausgeschlossen werden.

Bei Feststellung einer Knochenmarkfibrose bedarf es der Quantifizierung der Fibrose, wobei hier das einheitliche Grading nach Thiele [34] zu empfehlen ist.

\section{Zelluläre Knochenmarknekrose}

Zelluläre Nekrosen sind zum einen mögliche Nebenwirkungen myeloablativer Therapien. Zum anderen sind sie daneben stets ein Verdachtszeichen einer fokalen Infiltration durch ein Malignom und erfordern auf jeden Fall eine weiterführende Untersuchung, z. B. in tieferen Schnittstufen, zum Ausschluss fokaler neoplastischer Infiltrate ( $\bullet$ Abb. 8a), insbesondere Infiltrate akuter Leukämien [8].

\section{Seröse/gelatinöse Transformation des Knochenmarkstromas}

Dies ist ein spezifisches Reaktionsmuster des Knochenmarks mit Auftreten eines homogenen, agranulären und azellulären eosinophilen Materials zwischen den Fettzellen des Stromas ( $\bullet$ Abb. 8b). Das Material entspricht größtenteils Hyaluronsäure. Solche Veränderungen sind überdurchschnittlich häufig bei Malnutrition, insbesondere Anorexia nervosa, sowie bei AIDS und gelegentlich nach Chemotherapie aufzufinden [4]. In der Differenzialdiagnose müssen v. a. allem Amyloidosen in Betracht gezogen werden. Dabei helfen einfache histochemische Untersuchungen, da gelatinöse Transformationen alcianblaupositiv und Amyloidosen kongorotpositiv erscheinen.

\section{Veränderungen der Knochenmarkgefäße}

Veränderungen der Knochenmarkgefäße begleiten zahlreiche neoplastische und nichtneoplastische Prozesse des Knochenmarks. Praktisch alle Erkrankungen, die zu einer Faservermehrung im Knochenmark führen, werden durch eine Erhöhung der Mikrogefäßdichte begleitet. Das neoplastische Wachstum im Knochenmark ist ebenfalls mit einer Erhöhung bzw. Veränderung der Mikrogefäße assoziiert [27]. Nach Chemotherapie, insbesondere bei akuten Leukämien, nimmt der intramedulläre Druck ab, sodass das erhöhte Mikrogefäßnetzwerk dilatieren und gelegentlich kavernöse Hämangiome vortäuschen kann (• Abb. 8c, d).

\section{Fazit für die Praxis}

- Die Kenntnis der normalen Histologie des Knochenmarks sowie der häufigsten reaktiven Veränderung ist eine Grundvoraussetzung für die histopathologische Beurteilung ( $\bullet$ Infobox 1).

- Beim Knochenmark handelt es sich um das wahrscheinlich dynamischste Gewebe des Menschen, das ausgeprägte alters- und situationsabhängige morphologische Veränderungen aufweist.

- Die detaillierte Kenntnis dieser Veränderungen sowie die kritische Berücksichtigung einer vollständigen klinischen Information inklusive (Differenzial-)Blutbild sowie der Resultate von Zusatzuntersuchungen (Aspirationszytologie, Flowzytometrie, Zytogenetik, Molekulargenetik) ermöglichen die Vermeidung einer Fehlbeurteilung sowie eine korrekte und spezifische Diagnosestellung.

\section{Korrespondenzadresse}

Prof. Dr. A. Tzankov

Institut für Pathologie, Universitätsspital Basel

Schoenbeinstr. 40, 4031 Basel

Schweiz

atzankov@uhbs.ch

Danksagung. Die Autoren möchten sich bei Dr. Bertha Frisch für ihre Pionierarbeit bei der histologischen Knochenmarkuntersuchung bedanken.

Interessenkonflikt. Der korrespondierende Autor gibt für sich und seine Koautoren an, dass kein Interessenkonflikt besteht.

\section{Literatur}

1. Bain BJ (2001) Bone marrow trephine biopsy. J Clin Pathol 54:737-742

2. Bartakke S, Abdelhaleem M, Carcao M (2008) Valproate-induced pure red cell aplasia and megakaryocyte dysplasia. Br J Haematol 141:133 
3. Bataille R, Jégo G, Robillard N et al (2006) The phenotype of normal, reactive and malignant plasma cells. Identification of "many and multiple myelomas" and of new targets for myeloma therapy. Haematologica 91:1234-1240

4. Böhm J (2000) Gelatinous transformation of the bone marrow: the spectrum of underlying diseases. Am J Surg Pathol 24:56-65

5. Brown DC, Gatter KC (1993) The bone marrow trephine biopsy: a review of normal histology. Histopathology 22:411-422

6. Bruin M, Dassen A, Pajkrt D et al (2005) Primary autoimmune neutropenia in children: a study of neutrophil antibodies and clinical course. Vox Sang 88:52-59

7. Brunner A, Kantner J, Tzankov A (2005) Granulomatous reactions cause symptoms or clinically imitate treatment resistance in small lymphocytic lymphoma/chronic lymphocytic leukaemia more frequently than in other non-Hodgkin lymphomas. J Clin Pathol 58:815-819

8. Colvin BT, Revell PA, Ibbotson RM, Turnbull AL (1980) Necrosis of bone marrow and bone in malignant disease. Clin Oncol 6:265-672

9. Dokal I, Vulliamy T (2008) Inherited aplastic anaemias/bone marrow failure syndromes. Blood Rev 22:141-153

10. Engels K, Oeschger S, Hansmann ML et al (2007) Bone marrow trephines containing lymphoid aggregates from patients with rheumatoid and other autoimmune disorders frequently show clonal Bcell infiltrates. Hum Pathol 38:1402-1411

11. Felderbauer P, Ritter PR, Mattern D et al (2004) Acquired pure megakaryocytic aplasia: a separate haematological disease entity or a syndrome with multiple causes? Eur J Haematol 72:451-454

12. Foucar K, Reichard K, Czuchlewski D (2010) Bone marrow pathology. 3. Aufl. ASCP Press, Chicago

13. Gale E, Torrance J, Bothwell T (1963) The quantitative estimation of total iron stores in human bone marrow. J Clin Invest 42:1076-1082

14. Girodon F, Favre B, Carli PM et al (2001) Minor dysplastic changes are frequently observed in the bone marrow aspirate in elderly patients without haematological disease. Clin Lab Haematol 23:297-300

15. Gruppo RA, Lampkin BC, Granger S (1977) Bone marrow cellularity determination: comparison of the biopsy, aspirate, and buffy coat. Blood 49:2931

16. Harris AC, Todd WM, Hackney MH, Ben-Ezra J (1994) Bone marrow changes associated with recombinant granulocyte-macrophage and granulocyte colony-stimulating factors. Discrimination of granulocytic regeneration. Arch Pathol Lab Med 118:624-629

17. Hassanein NM, Alcancia F, Perkinson KR et al (2009) Distinct expression patterns of CD123 and CD34 on normal bone marrow B-cell precursors ("hematogones") and B lymphoblastic leukemia blasts. Am J Clin Pathol 132:573-580

18. Ibáñez L, Vidal X, Ballarín E, Laporte JR (2005) Population-based drug-induced agranulocytosis. Arch Intern Med 165:869-874

19. Janka GE (2007) Hemophagocytic syndromes. Blood Rev 21:245-253

20. Kirby MA, Weitzman S, Freedman MH (1990) Juvenile chronic myelogenous leukemia: differentiation from infantile cytomegalovirus infection. Am J Pediatr Hematol Oncol 12:292-296
21. Krause JR, Penchansky L, Knisely AS (1992) Morphological diagnosis of parvovirus B19 infection. A cytopathic effect easily recognized in air-dried, formalin-fixed bone marrow smears stained with hematoxylin-eosin or Wright-Giemsa. Arch Pathol Lab Med 116:178-180

22. Kuter DJ, Bain B, Mufti G et al (2007) Bone marrow fibrosis: pathophysiology and clinical significance of increased bone marrow stromal fibres. Br J Haematol 139:351-362

23. Lu W, Uetrecht JP (2008) Peroxidase-mediated bioactivation of hydroxylated metabolites of carbamazepine and phenytoin. Drug Metab Dispos 36:1624-1636

24. Marsh JC, Ball SE, Cavenagh J et al and British Committee for Standards in Haematology (2009) Guidelines for the diagnosis and management of aplastic anaemia. Br J Haematol 147:43-70

25. McMillan R (2007) The pathogenesis of chronic immune thrombocytopenic purpura. Semin Hematol 44(Suppl 5):3-11

26. Medinger M, Buser A, Stern M et al (2012) Aplastic anemia in association with a lymphoproliferative neoplasm: coincidence or causality? Leuk Res 36:250-251

27. Medinger M, Fischer N, Tzankov A (2010) Vascular endothelial growth factor-related pathways in hemato-lymphoid malignancies. J Oncol 729725

28. Naeim F (1995) Topobiology in hematopoiesis. Hematol Pathol 9:107-119

29. Poje EJ, Soori GS, Weisenburger DD (1992) Systemic polyclonal B-immunoblastic proliferation with marked peripheral blood and bone marrow plasmacytosis. Am J Clin Pathol 98:222-226

30. Qubaja M, Marmey B, Le Tourneau A et al (2009) The detection of CD14 and CD16 in paraffin-embedded bone marrow biopsies is useful for the diagnosis of chronic myelomonocytic leukemia. Virchows Arch 454:411-419

31. Rimsza LM, Larson RS, Winter S (2000) Benign hematogone-rich lymphoid proliferations can be distinguished from B-lineage acute lymphoblastic leukemia by integration of morphology, immunophenotype, adhesion molecule expression, and architectural features. Am J Clin Pathol 114:66-75

32. Rosenthal NS, Farhi DC (1989) Bone marrow findings in connective tissue disease. Am J Clin Pathol 92:650-654

33. Sawada K, Hirokawa M, Fujishima N (2009) Diagnosis and management of acquired pure red cell aplasia. Hematol Oncol Clin North Am 23:249-259

34. Thiele J, Kvasnicka HM, Facchetti F et al (2005) European Consensus on grading bone marrow fibrosis and assessment of cellularity. Haematologica 90:1128-1132

35. Thiele J, Ammers E von, Wagner S et al (1991) Megakaryocytopoiesis in idiopathic thrombocytopenic purpura: a morphometric and immunohistochemical study on bone marrow biopsies with special emphasis on precursor cells. Hematol Pathol 5:75-82

36. Thiele J, Zirbes TK, Kvasnicka HM, Fischer R (1999) Focal lymphoid aggregates (nodules) in bone marrow biopsies: differentiation between benign hyperplasia and malignant lymphoma - a practical guideline. J Clin Pathol 52:294-300

37. Valent $P$ (1995) Mast cell differentiation antigens: expression in normal and malignant cells and use for diagnostic purposes. Eur J Clin Invest 25:715720
38. Wang EC, Borysiewicz LK (1995) The role of CD8+, CD57+ cells in human cytomegalovirus and other viral infections. Scand J Infect Dis Suppl 99:69-77

39. Wickramsinghe SN (2007) Bone marrow. In: Mills SE (Hrsg) Histology for pathologists. Lippincott Williams \& Wilkins, Philadelphia, S 799-836

40. Wilkins BS (2011) Pitfalls in bone marrow pathology: avoiding errors in bone marrow trephine biopsy diagnosis. Review. J Clin Pathol 64:380-386 